\title{
Light-weight and Low-cost HealthCare - A Cloud based BAN for Remote Health Monitoring
}

\author{
${ }^{1}$ Teena Hinesh Bachani, ${ }^{2}$ Himesh Kumar, ${ }^{3}$ Sana Hoor Jokhio, ${ }^{4}$ Imran Ali Jokhio \\ ${ }^{1}$ IICT MUET Jamshoro, Pakistan, \\ ${ }^{2}$ IBET LUMHS University Jamshoro, Pakistan \\ ${ }^{3}$ Dept. of CSE MUET Jamshoro, Pakistan, \\ ${ }^{4}$ School of Information Tech. \& Eng., Melbourne Institute of Technology, Melbourne, \\ Australia \\ ${ }^{1}$ teenakumari18@hotmail.com, ${ }^{2}$ hinesh.kumar@lumhs.edu.pk, ${ }^{3}$ sana.hoor@faculty.muet.edu.pk, \\ 4ijokhio@mit.edu.au
}

\begin{abstract}
A BAN, which fundamentally comprises of remote wearable sensor nodes typically organized by means of a sta-tionary or portable device, is principally adopted to examine single aided livings. A system of BANs worn by a group of individuals for continuous monitoring delivers huge sum of contextual information that require an adaptable, scalable and cost effective approach for storage capacity as well as elaboration. In this paper, we propose a cloud-integrated light-weight and low-costs health care wireless BAN. Our proposed system is intended to evaluate and observe significant physiological information of a patient with a specific end goal to precisely portray the status of her/his wellbeing and wellness anytime anywhere. To ensure accuracy and reliability the proposed BAN has been field tested. The test outcomes demonstrate that it is able to calculate the patient's physiological vitals with a very high accuracy.

Index Terms-Wireless Body Area Network, Cloud Comput-ing, Smart Health Care, Remote Patient monitoring system
\end{abstract}

\section{INTRODUCTION}

A promising technology nowadays in the medical field is the wireless body area networks (WBANs). WBAN com-prises of a range of small scaled or miniaturised sensor nodes that are portable and autonomous in nature. These may be worn for patient's long-term health monitoring by continuously collecting health data and/or information of the patients. Nevertheless, these WBANs can be a good source of managing catastrophic events, performance of rescue teams and effectiveness in health monitoring [1]. The sensor nodes that are part of a WBAN, collect huge amount of data which give rise to the challenges such as on-demand, scalable, efficient processing and secure storage infrastructure [2]-[4]. Cloud computing may be considered to play a vital role in accomplishing the above objectives. Within the environment of a cloud various devices may be linked together that may range from small-scale sensor devices to super computers for managing and delivering services to hospitals, industries or loved ones. The integration of cloud computing with WBAN may produce possible hybrid platform 


\section{Gyancity Journal of Engineering and Technology, Vol.4, No.1, pp. 19-30, January 2018 \\ ISSN: 2456-0065 DOI: 10.21058/gjet.2018.41003}

that can process huge amount of data collected from various WBAN sources. Hence the system may enable physicians, nurses and even loved ones to access the storage and processing infrastructure globally at anytime. WBAN enables health parameters to be monitored continuously and remotely and of course in real-time. The data may then be processed and accessed or transferred to medical facilities and their databases [5]. Staff of a healthcare organization, insurance companies and government agencies may be sharing the collected data for various reasons and purposes. Moreover, during a disaster, at the scene, people may greatly benefit from such technologies that are used for contin-uous monitoring of their vitals and tracking their whereabouts until they are admitted to some facility. The welfare system of many countries suffers economical support especially with respect to medical and health benefit claims, etc. With the help of WBANs the overall cost of hospitalization may be reduced for patients. This gives the opportunity to patients to be remotely monitored from home or some special/dedicated environments. Such systems may play an important role in reducing the financial load of an individual as well as the healthcare [6]. Medical sensing devices may be deployed in such environments to collect the physiological parameters of the individual under monitoring and detect an anomaly as early as possible to health professionals in various facilities. Moreover, with the help of cloud computing/technology the expensive health management software may be outsourced to the cloud and the OPEX costs may be reduced but at the same time increasing the usage to such a level to decrease the overall cost per patient significantly. BAN is predicted to give a free rein to a wave of personalized, novel and incorporated medical, standard of living, gaming, fitness, entertainment, military and consumer electronics applications. Wireless connectivity of body sensor network make it possible to implant drug delivery, implant cardioverter-defibrillator (ICD), swallow camera pills, wearable ECG/EMG/ EEG/BP/SpO2/temp monitoring, sleep analysis, gait analysis, high risk pregnancy monitoring, media players, headsets and emotion detection [7]. Figure below demonstrates few of the applications which BAN technology is wished to support. Combining the BAN, cloud computing and high-speed internet can help various health personnel may be able to remotely manage their patients more efficiently and in a costeffective manner. However, not to forget the constraints of WSN that may affect the overall lifetime of the medical sensing devices and their operations significantly. This research mainly focuses on how to provide a light-weight and low-cost remote unobtrusive long-term patient monitoring service with real-time updates to health care institutes and individuals. The idea is to merge the information processing 


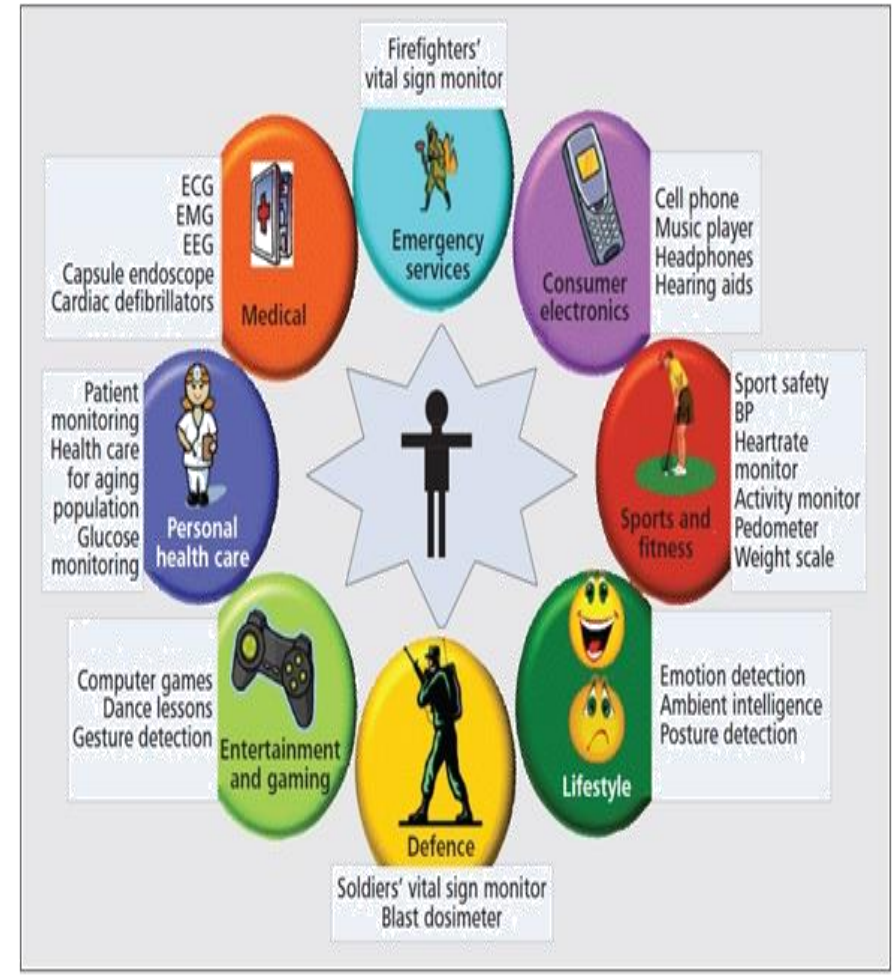

Fig. 1. WBAN Applications [7].

technologies and advances in communication (via using cloud computing and BAN) to provide cost-effective and efficient solutions. This research will highlight need for the integration of cloud computing with Wireless Body Area Networks, cloud services for WBANs, data storage and distribution. The research encompass reducing the communication overhead issues in WBANs by development of a real-time light-weight remote sHealth Care system using medical sensors to monitor remote patients. This paper has been structured into 5 Sections. Section 2 discusses related work including some globally proposed health care systems. Some distinguished features of WBANs i.e. its issues and challenges, role of cloud computing integrated WBAN in health care system and critical factors are discussed in Section 3. Section 4 elaborates requirements, necessities, design, implementation and results of our proposed system. Finally we conclude our findings in Section 5 along with some directions for future research.

\section{RELATED WORK}

The integration of IoT with Cloud Computing is grabbing the attention globally and researchers have been investigat-ing and analysing various aspects of it over the past few years. Based on the resources and service types which are offered via the cloud, Cloud Computing is adopted to offer Software as a Service (SaaS) by linking objects by means of the internet [8]. Cloud Computing deals with the mecha-nism to stock up diverse data 


\section{Gyancity Journal of Engineering and Technology, Vol.4, No.1, pp. 19-30, January 2018 \\ ISSN: 2456-0065 DOI: 10.21058/gjet.2018.41003}

apart from its coverage area, where as on other end IoT manages to provide systematic events to be executed for any device via internet. Therefore, introducing Cloud Computing and IoT together can lead towards improved capacity which can be rapidly enhanced without putting on further more infrastructure investments. IoT solution deployed with Cloud technology in healthcare affects on both figures of time and cost. Therefore it can be said that when undeveloped and surviving countries are facilitated with such type of combo solutions then they can avail healthcare services in real-time, remotely and instantly [9]-[12]. Nowadays, some intense research is being carried out in the domain of pervasive healthcare to make e-health services much more better; however, some of these use cloud technology as a latest IT paradigm, which are reviewed here. Research work of F.A Khan et Al., [15] is twofold: firstly, it aims to secure communication between the sensors in WBANs that is achieved by a key generation scheme based on multi-biometric and then, it focuses on preserving the privacy of patient's data i.e. (EMRs) medical records electronically stored in the community cloud of hospital. The incentive behind the practice of multiple biometrics is to lengthen, and acquire a more secure and random key. Multi-biometric scheme is basically utilizing the merging of two biometrics consisting couple of physiological values i.e. electroencephalogram EEG and electrocardiogram ECG. For a secure communication, features are extracted and quantized and then using testing suite of Dieharder, i.e. a wellknown randomness-testing suite, keys generated are verified. The other part is about the privacy of patients' data store over cloud. So for that author, based on dynamic reconstruction of metadata, has adapted a pri-vacy preservation mechanism. Data classification is performed followed by the table splitting. The prototype is assessed in terms of security of inter-sensor communication yielding a worthwhile solution for the next generation journey of remote healthcare systems. However, upcoming work is needed in particular to sustain tough security in a real-time medical care application; privacy and security needs to be included from the initial stage of application design analysis up to execution. Suraj Pandey et Al., [16] proposed a Cloud environment which is autonomic in nature, gathers an individual's health information and stocks it to a Cloud-based data warehouse facilitating data mining by means of software services offered in the Cloud. A prototype system is developed, collecting electrocardiogram (ECG) data from volunteers acquired in real-time to carry out analysis of ECG beats for evaluation. This system is hosted as a web-service where Platform as a Service layer manages the execution of the software system via three chief components: (i) Workflow Engine, (ii) Container scaling manager, and (iii) Aneka. The workflow engine resid-ing within a container; deals with the execution of workflow tasks of the ECG application. As user requests increase, so to deal out with every request to workflow engines, more containers are instantiated by container scaling manager. From there the tasks created by user requests are submitted to Aneka. Aneka controls the communication between the $\mathrm{PaaS}$ and IaaS layer by means of a master-worker framework. Where the QoS is maintained by the 'Dynamic Scalable Runtime" (DSR) module i.e. part of Aneka scheduling environment. Author suggested for a trusted authority to develop an authentication and authorization system called Public Key Infrastructure (PKI) based. PKI helps in the encryption/decryption of any information exchanged between the 


\section{Gyancity Journal of Engineering and Technology, Vol.4, No.1, pp. 19-30, January 2018 \\ ISSN: 2456-0065 DOI: 10.21058/gjet.2018.41003}

middleware and applica-tion layer solving scalability and cost issues in a non-disruptive manner. But the results of the prototype and the experiments depict, this security infrastructure is not utilized here. In future cost minimization may be achieved by exploring Heuristic-based techniques using Cloud services, while maintaining QoS satisfaction to users. Also techniques to tackle data security problems when using distributed Cloud storage need attention in future. A. Lounis et Al., [17] introduces Cloud based architecture for medical wireless sensor networks with the assurance of the secrecy and the reliability of outsourced health status data excluding involvement of patients or doctors. Also an innovative access control is proposed allowing the implementation of security policies that are complex and dynamic in behaviour necessary for medical application re-ducing the management and processing overhead. To achieve fine-grained access control, Ciphertext Policy Attribute Based Encryption (CPABE) is combined with symmetric encryption. To tackle the complexity problem of security management, an entity called Healthcare Authority (HA) is introduced which details and imposes the security policies of the health-care organization. The proposed solution indicates through preliminary performance evaluation that the time required for encryption/decryption and the management overhead is reduced. Results indicated control access scheme is more able in terms of cryptographic operations. But since work has been done in different units separately hence a complete and comprehensive performance assessment is needed in upcoming time. Whereas distributed attribute-based encryption to have multi healthcare authorities may need to be addressed as one of the future open research issue. K. Murugalakshmi et Al., [18] discusses present advances in medical care systems with cloud computing technology to examine heart rate, temperature, and the energy efficient routing as well as QOS factors with focus on applications for head and neck injuries. The system has sub-sequent modules: Dataset Collection, Android User Interface, Cloud Storage, Monitoring the data and integrating it with a source system. In real time environment the sensors attached to the microcontroller, from microcontroller associated to the android application (i.e.) human vital signs application via the Bluetooth communication. The remaining work (i.e.) storing to the cloud, Monitoring the data and interfaces with a source system will be implemented in the future work. Bhoomika and Muralidhara [19] presented the idea for IOT based smart healthcare system over internet. Hence the solution provides real time observation of the health care vitals to medical practitioners/doctors using sensors like pulse oximeter sensor and temperature sensor and Microcontroller PIC18F46K22. To alert the caretaker about deviation in sensor results, a buzzer is also connected. Password protected ESP8266 Wi-Fi module, encrypted by standard AES128 addresses the privacy and secrecy matter. In the developed system the improvement would be adding more sensors to the system measuring various other valuable parameters beneficial for patient monitoring and connecting with internet for quick and easy access. To increase the range of communication, setting up a Wi-Fi mesh type network to would be an increment. Balamurugan and Ajay [20] proposed an "infrastructure-independent" model of health care, with cloud computing services to provide and prompt appropriate treatment of casualties, in some cases prevent additional casualties. The system aims to measure various vital physiological health parameters like ECG, body temperature,Fall Detection, EEG and Blood Pressure etc., of in real-time and 


\section{Gyancity Journal of Engineering and Technology, Vol.4, No.1, pp. 19-30, January 2018 \\ ISSN: 2456-0065 DOI: 10.21058/gjet.2018.41003}

transfer his/her health parameters wirelessly using Zigbee, to a remote base station referred as Local Processing Unit[LPU].Future work is required in the direction of design of a novel intra-body biosensor for intra-vaginal temperature monitoring, as the precise measure of core body temperature data is highly desired in various medical applications. Hence an intra-body biosensor is a necessity. IBM Research Division [21] proposed a new infrastructure called Sensor- Cloud in-frastructure in which physical sensor is virtualized as a virtual sensor on the cloud computing. It is a move towards enabling the management of sensors over cloud in which virtual sensors grouped dynamically on cloud computing can be automatically provided on user demand without any concern of their locality and detailed specifications. The users can employ virtual sensors and control them with standard functions. Various sensors with multiple owners can come up on Sensor-Cloud in-frastructure. This infrastructure is quite advantageous in terms of implementing Virtualization, Standardization, Automation, and Monitoring, Grouping of the virtual sensors and their implementation as the service models. But for implementation of this infrastructure some IT resource must be prepared. Also SensorCloud administrators need to prepare the templates for virtual sensors so these both necessities are not crucial in direct sharing physical sensors mechanism. Author divided this work into sensor system management and sensor data management. But this approach focuses only on management of sensor system. So if sensor data management mechanism is also integrated with this, higher quality services may be expected. $\mathrm{Xu} \mathrm{Wu}$ [22] introduced a lightweight trust-based access control model, in which on the basis of the trust value in the trust certificates and role of the user, the users can have their access control privileges for the electronic healthcare information. The user trust value in the certificate can be com-puted by a user behaviour-based trust scheme. This method can attain identity authorization and authentication simultaneity in a cloud-based WBAN. Hence it can better alleviate the energy consumption problem. But in crafting a protected wMCC platform, several design aspects including encryption, flexibility, access rights to control, data fragmentation, user assortment, and portable access must be taken in consideration. Communication from user to RIDB and TMS Server lead to-wards communication overhead hence further improvement in context of communication overhead is needed. In system that is intended for continuous monitoring of patients remotely can be overwhelming if performed partially manually; so for that reason a huge level of automation is mandatory which can be helpful from the collection and sensing of vital information up to the safe and secure access to the concerned people/services. Our proposed work provides a light-weight as well as low-cost solution for monitoring patient's vitals remotely that is affordable as well as implementable for countries possessing low economic welfare. Since we are in very much need of cost effective solution for remote patients; who cannot meet the high prices of expensive medical devices, Hence for that an economic prototype that works from gathering data of patient's 


\section{Gyancity Journal of Engineering and Technology, Vol.4, No.1, pp. 19-30, January 2018 \\ ISSN: 2456-0065 DOI: 10.21058/gjet.2018.41003}

vitals to make it available to care providers/doctors has been developed. We discuss the detailed design and implementation of our proposed prototype in the next section.

\section{PROTOTYPE DESIGN AND IMPLEMENTATION}

Body Area Sensor network is an exclusive type of ar-rangement designed and developed to observe, handle and correspond with various health parameters of an individual like Blood pressure, temperature and ECG etc [13], [14]. The health parameters can be examined by number of sen-sors deployed on the body else clothing or even beneath an individual's skin. Any type of drug may be infused by means of actuator set up in the individual's body so as to control the temperature or Electrocardiographic signals or any life-saving medicines. Main Central unit is in charge to set up correspondence among sensors, actuators and mobile phones wirelessly. Cell phone or Computer may be helpful in passing the information between a person's bodies to the external world (physician, emergency). BANs play a vital role in providing updated and timely information to health care systems. This information may be used to save precious lives of people under observation locally and/or remotely. We propose a smart health care system (LLsHC) prototype that may be used for remote monitoring of patients to help the carers and doctors take timely decisions in critical situations. This section discusses the design and development of our proposed prototype in detail.

\section{A. Interfacing and Programming of sensor nodes}

This step manifested the beginning of hardware perception of the prototype. Initially, the sensors were tested to check their compatibility with the human body. The response of the stand-alone sensor determined whether any additional conditioning circuitry was required or not. All the sensors after their individual testing were interfaced with an Arduino controller. This step covered the programming of the Arduino controller to collect the biometric data and convert it into a form suitable for wireless transmission. There are two bio-medical sensors used in this prototype which are hard-wired to the Arduino controller. The whole arrangement acts as a single sensor node. For two different patients two different sensor nodes are used while on a single senor node multiple sensors can be attached, according to the need of the patient. The data from Arduino will be sent, which act as central hub with the help of wireless transmission by "ESP8266" attached to the Arduino. Arduino with the help of "ESP8266" module and upload the sensor data on the database server named "Firebase" in real time. But how the components are being connected step by step to execute properly is a crucial point to consider. The main reason to use ESP8266 module instead of Arduino Wi-Fi Shields or any other Wi-Fi module is that it is easier and a cost-effective alternative component as compare to others. All ESP8266 modules are easily programmable, provided with default factory firmware. User can also write its own firmware and upload into its module. The default firmware provides a serial communication which can be used to send commands to the module to connect with any Wi-Fi router and transmit/receive data and can be easily integrated with Arduino. All the individual nodes were connected wirelessly to a data-aggregate node. The mode of wireless communication selected in the first stage. The 


\section{Gyancity Journal of Engineering and Technology, Vol.4, No.1, pp. 19-30, January 2018 ISSN: 2456-0065 DOI: 10.21058/gjet.2018.41003}

transmitter and receiver used for the wireless communication were connected to the individual nodes and the controller. The controller processed the biometric data to determine the critical levels. If a message is received from the sensor nodes depicting a critical level then the alarm/alert system is required to be triggered. The data-aggregate node will also be responsible for sending the data periodically to a nearby healthcare system. This stage proved to be the most challenging of all, as it covers the topics we have limited experience with. The programming of the controller and data aggregation also took place during this stage. The components of a data aggregate node are as follows:

1) Arduino UNO

2) Temperature Sensor IC LM35

3) Spo2 Sensor

4) ESP8266

5) Firebase Cloud

\section{B. Software Development}

The data aggregated in the previous stage was manipulated to give meaningful visual conclusions. This was achieved by the implementation of a web-based application which displayed the data collected in each neighbourhood in real-time. This software will especially be useful for remote healthcare monitoring where a remotely located patient can be monitored by qualified health personnel without any need of physical interaction. Database is used for remote and long-lasting storage of useful data. The data aggregate node has limited memory and it can't keep the data acquired by various patients forever. Thus a reliable storage location which is not physically located on the data aggregate node is essential. Initially, we considered using MySQL server to create a local database and access it from a remote web application.

1) Unit Testing of Hardware: This test incorporates testing each and every component specified in the equipment exe-cution. These tests are carried out individually first before interfacing another part.

Sensors

Temperature Sensor IC LM35(Shows a multimeter read-ing corresponding to $0.1 \mathrm{~V} / 10 \mathrm{C})$

SPO2Sensor (Shows $95 \%$ to $100 \%$ blood oxygen level on Arduino Serial Monitor)

Arduino - Assured programs correctly, reads analog pins/Properly Boots Up and read GPIO Pins

ESP8266 Wi-Fi Module - Checked Microcontroller has the ability to talk to a TTL serial device.

2) System Testing of Hardware: After successful execution of unit testing, it is confirmed that all the components are working properly hence all the circuitry can be integrated to-gether in to a single hardware system and finally system testing is performed. Figure below shows hardware components inte-grated into a complete system. Temperature and SPO2sensor 


\section{Gyancity Journal of Engineering and Technology, Vol.4, No.1, pp. 19-30, January 2018 \\ ISSN: 2456-0065 DOI: 10.21058/gjet.2018.41003}

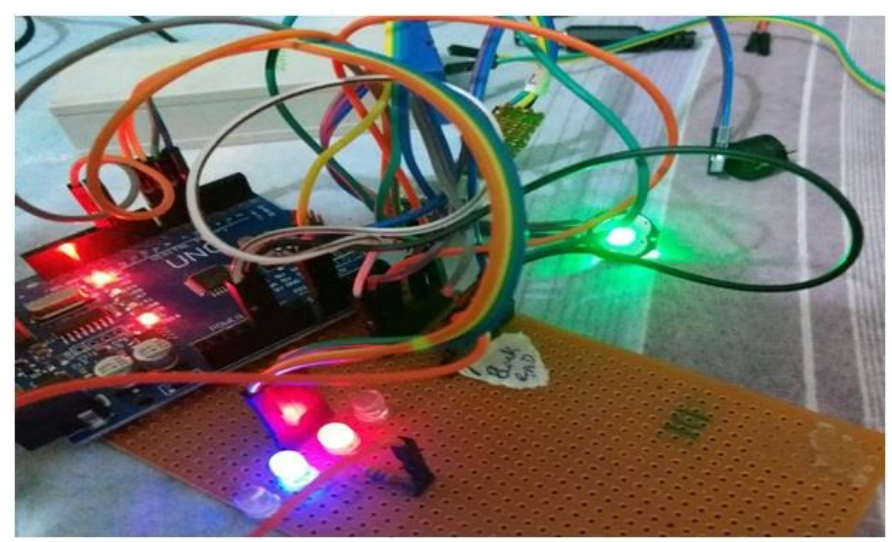

Fig. 2. Hradware system of prototype.

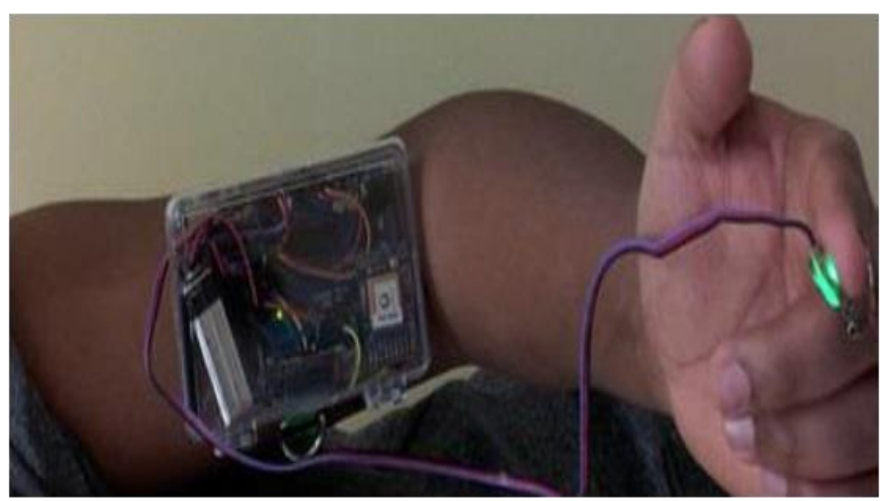

Fig. 4.Arduino Testing.

were hooked up to the Arduino and their readings were observed through Arduino serial monitor. The screenshots of temperature and SPO2 sensor readings are shown in the figure below. The purpose of the Arduino test was to verify that the

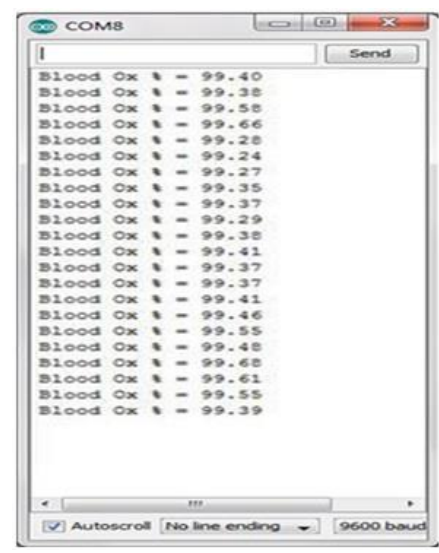

Fig. 3. SPO2 and Temperature sensor reading results.

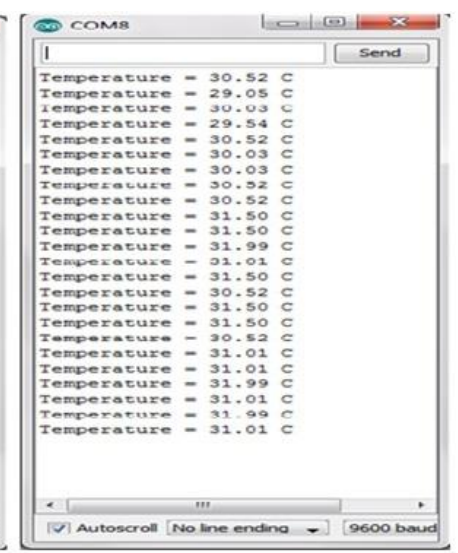

programming pins setup and allowed for the microcontroller to be programmed. This was tested by flashing a simple blink LED program. The setup for the programme was found to be correct. The analog pin was tested by applying a sine wave via a function generator to the pin and observing the output in Arduino Serial Monitor. This test verified the working of the analog pins of Arduino Microcontrollers. Software tests are done to verify the correct working of the RF code, the Python and database uploading code, web pages, Firebase Scripts etc. Every test was completed effectively and their outcomes may be observed via the accompanying figures. RF sniffer has been verified during the hardware tests. Below are some of the snapshots of software prototype of database created to collect, view and transmit the data. Figure below shows records of two patients generated i.e. patient 1 and patient 2. Information originated with the aid of a BAN may be processed continuously by the BAN controller in real-time and/or 
potentially transmitted to a server-side for long term storage and online/offline operations. Hence, a cloud integrated WBAN may provide a flexible processing infrastructure and storage to carry out both online and offline examination of data streams of body sensors. The prototype was successfully implemented and tested. However, implementing the designed prototype in a real scenario and on large scale may depend on a number of issues and challenges that need to be analyzed.

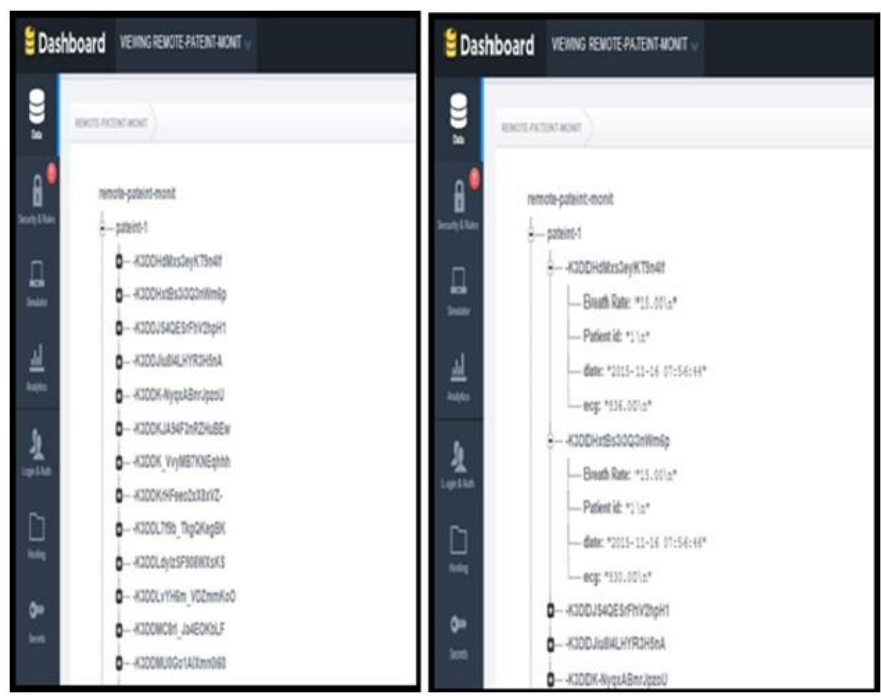

Fig. 6. Records in Database.

\section{CONCLUSION}

A lightweight and low-cost sHealthCare System prototype has been proposed in this paper. The aim of this research was to explore the integration of Body sensor network and cloud computing technology towards the betterment of human lifestyle while the patient is at a remote site. A thorough discussion on the existing approaches of the WBAN as in the healthcare system have also been presented in this paper. Cloud computing is doubtlessly an interesting issue inside the business world. Since it is a moderately latest innovation, its functioning along with wireless network of sensors involves attentive attention. while developing this prototype the aim was to find and analyse the variables associated with the real-time BAN and its functionalities. Our proposed prototype implementation showed promising results. Hence, we would like to explore our proposed prototype further. As our future work we would like to extend our prototype design to include remote access to patient health records for doctors so that in case of an emergency the medical details can be used to treat the patients more effectively. Nevertheless, a number of issues need attention with respect to the Cloud IoT environment. Light weight efficient usage of sensing devices, end-to-end data delivery, resource management, etc., are some challenges that need to be focused. Moreover, the combination of cloud, data mining, IoT and WBAN is an interesting new field to be explored in detail to help the medicine industry help humanity in a true manner. 


\section{Gyancity Journal of Engineering and Technology, Vol.4, No.1, pp. 19-30, January 2018 \\ ISSN: 2456-0065 DOI: 10.21058/gjet.2018.41003}

\section{REFERENCES}

[1] R. Cavallari, F.Martelli,R.Rosini, C. Buratti, and R.Verdone, "A survey on wireless body area networks: technologies and design challenges," IEEE Communications Surveys and Tutorials, vol. 16, no. 3, pp. 1635- 1657, 2014.

[2] W. Y. Toh, Y. K. Tan, W. S. Koh, and L. Siek, Autonomous wearable sensor nodes with flexible energy harvesting, IEEE Sensors Journal, vol. 14, no. 7, pp. 2299-2306, 2014.

[3] P. Pillatsch, E. M. Yeatman, and A. S. Holmes, A wearable piezoelectric rotational energy harvester, in Proceedings of the IEEE International Conference on Body Sensor Networks (BSN '13), pp. 1-6,May 2013.

[4] J. Wan, C. Zou, S. Ullah, C.-F. Lai, M. Zhou and X. Wang, "Cloud- Enabled Wireless Body Area Networks for Pervasive Healthcare," IEEE Network, vol. 27, no. 5, (2013), pp. 56-61.

[5] S. Chatterjee, A. K. Das and J. K. Sing, "A novel and efficient user access control scheme for wireless body area sensor networks," Journal of King Saud University-Computer and Information Sciences, Available online, (2013) October 26.

[6] M. Wen, J. Lei, J. Li, Y. Wang and K. Chen, "Efficient user access control mechanism for wireless multimedia sensor networks", Journal of Computational Information Systems, vol. 7, no. 9, (2011), pp. 3325- 3332 .

[7] A. Tewari, P. Verma, "Security and Privacy in E-Healthcare Monitoring with WBAN: A Critical Review", International Journal of Computer Applications (0975 - 8887) Volume 136 - No.11, February 2016.

[8] D. M. Barakah, M. Ammad-uddin," A Survey of Challenges and Applications of Wireless Body Area Network (WBAN) and Role of A Virtual Doctor Server in Existing Architecture", Third International Conference on Intelligent Systems Modeling and Simulation,2012.

[9] S.A. Shah, S.M.K Raazi, R.A. Khan," Wireless Senor Networks Health Monitoring: Trends and Challenges", Journal of Emerging Trends in Computing and Information Sciences", VOL. 3, NO. 3, March 2012.

[10] N.B. Roy and D. Das," Role of Body Area Sensor Networks in Smart Health Care”, International Journal of Future Computer and Communication", Vol. 4, No. 5, October 2015.

[11] N. Alharbe, A. S. Atkins,U. Champion," Use of Cloud Computing with Wireless Sensor Networks in an Internet of Things Environment for a Smart Hospital Network", The Seventh International Conference on eHealth, Telemedicine, and Social Medicine, eTELEMED 2015

[12] J.M. L. P.Caldeira, J. J. P. C. Rodrigues, and P. Lorenz, "Toward ubiquitous mobility solutions for body sensor networks on healthcare," IEEE Communications Magazine, vol. 50, no. 5, pp. 108-115, 2012.

[13] E. Ibarra, A. Antonopoulos, E. Kartsakli, J. J. P. C. Rodrigues, and C. Verikoukis, ”Joint power-QoS control scheme for energy harvesting body sensor nodes," in Proceedings of the IEEE International Conference on Communication (ICC'14), pp. 3511-3516, 2014.

[14] S. Poorejbari, H. Vahdat-Nejad, "An Introduction to Cloud-Based Per- vasive Healthcare Systems."

[15] F.A. Khan, A. Ali, H. Abbas and N.H. Haldar," A cloud-based healthcare framework for security and patients' data privacy using wireless body area networks", The 2nd International Workshop on Communications and Sensor Networks (ComSense-2014).

[16] W. Voorsluys S. Niu, R. Buyya and A. Khandoker, "An Autonomic Cloud Environment for Hosting ECG Data Analysis Services".

[17] A. Lounis, A. Hadjidj, A.Bouabdallah and Y. Challal,"'Secure and Scal- able Cloud-based Architecture for e-Health Wireless Sensor Networks", International Conference on Computer Communication Networks (IC- CCN), Jul 2012, Munich, Germany. pp.1, 2012. ¡hal-00695956i.

[18] K. Murugalakshmi , Mrs. G. Geetha and Mrs. K. Sundara Velrani

,"Cloud based healthcare monitoring system using wearable Sensors", International Journal of Emerging Technology in Computer Science and Electronics (IJETCSE) ISSN: 0976-1353 Volume 21 Issue 3 APRIL 2016.

[19] K N Muralidhara, Bhoomika .B.K ,"Secured Smart Healthcare Mon- itoring System Based on Iot", 


\section{Gyancity Journal of Engineering and Technology, Vol.4, No.1, pp. 19-30, January 2018 \\ ISSN: 2456-0065 DOI: 10.21058/gjet.2018.41003}

International Journal on Recent and Innovation Trends in Computing and Communication ISSN: 23218169 Volume. 3 Issue. 74958 - 4961.

[20] Balamurugan, Ajay, " Cloud Care: A Remote Health Monitoring Sys-tem"

[21] M. Youriyama, T. Kushida, "Sensor-Cloud Infrastructure Physical Sen- sor Management with Virtualized Sensors on Cloud Computing", IBM Research - Tokyo, IBM Japan, Ltd. 1623-14 Shimotsuruma, Yamato Kanagawa 242-8502, Japan .

[22] Xu Wu, "A Lightweight Trust-based Access Control Model in Cloud-Assisted Wireless Body Area Network" , International Jour- nal of Security and Its Applications Vol.8, No.5 (2014), pp.131-138 http://dx.doi.org/10.14257/ijsia.2014.8.5.13.

[23] D. M. Barakah, D.Ammad-uddin "A Survey of Challenges and Ap- plications of Wireless Body Area Network (WBAN) and Role of A Virtual Doctor Server in Existing Architecture", 2012 Third International Conference on Intelligent Systems Modelling and Simulation.

[24] S.Khan, Al-Sakib Khan Pathan, N.A. Alrajeh, Book "Wireless Sensor Networks: Current Status and Future Trends", November 16, 2016, Pages-171. 